\title{
SABANAS CULTIVADAS COMO SUMIDEROS DE CARBONO EN UN SUELO ÁCIDO-ARENOSO DE BAJA FERTILIDAD NATURAL
}

\author{
OJeda Falcón, A. D. ${ }^{1}$ \& LÓPeZ HeRnÁndeZ, D. ${ }^{1}$
}

\begin{abstract}
RESUMEN
Un Arenic Paleustults de sabana fue cultivado con especies adaptadas y fertilizado con dosis únicas de 1,0 y $0,3 \mathrm{Mg} \mathrm{ha}^{-1}$ de roca fosfórica (RF) y NPK, respectivamente. Un muestreo aleatorio de suelo, en los tratamientos, sabana natural (SN) y sabana cultivada (SC), a las profundidades de 0-3,0; 3,1-6,0 y 6,1-10,0 cm, permitió las determinaciones de textura, COS, fracción ligera de la materia orgánica del suelo (FL MOS), pHagua, $\mathrm{pH}_{\mathrm{KCl}}$, densidad aparente (Da), contenidos totales de nitrógeno $(\mathrm{N})$ y fósforo $(\mathrm{P}), \mathrm{P}$-disponible y bases intercambiables. Los análisis estadísticos incluyeron: ANOVA, ACP y un análisis de ruta, que generó modelos estadísticos multicausales. La SC mostró cambios significativos al acumular $2.970 \mathrm{~kg} \mathrm{ha}^{-1}$ de COS y presentar una densidad de carga neta coloidal (DCNC) 50\% más electronegativa, que se relacionó con la FL MOS, el COS y la Da, en contraste con la SN, cuyos factores de variación fueron de naturaleza inorgánicas.
\end{abstract}

Palabras clave: sabanas, sumidero de carbono, punto isoléctrico, modelos estadísticos, agricultura sustentable.

\begin{abstract}
Cropped Savannahs as carbon sink in acid sandy soils of low natural fertility.

An Arenic Paleustults savannah was cropped with adapted species and fertilized with unique doses of 1,0 and $0,3 \mathrm{Mg} \mathrm{ha}^{-1}$ of phosphoric rock and NPK, respectively. Random sampling of the treated soils at three depths $0-3,0 ; 3,1-6,0$ and $6,1-10,0 \mathrm{~cm}$ allowed the determina-
\end{abstract}

1.- Laboratorio de Agroecología. Centro de Ecología Aplicada. Instituto de Zoología y Ecología Tropical. Facultad de Ciencias. Universidad Central de Venezuela. Apdo. 47058. Caracas 1041-A. Venezuela. Teléfono: +58 416 6144451. Email: alonso.ojeda@gmail.com Manuscrito recibido el 5 de abril de 2019 y aceptado para su publicación el 12 de noviembre de 2019.

Ojeda-Falcón, A. D. \& López-Hernández, D. Sabanas cultivadas como sumideros de carbono en un suelo ácido-arenoso de baja fertilidad natural. FAVE - Ciencias Agrarias 18 (1): 25-44 CC BY-NC-SA 4.0 (c))(1)(5)(2) 


\section{A. D. Ojeda-Falcón et al.}

tion of: soil organic carbon (SOC), light fraction of the soil organic matter (LF SOM), total nitrogen $(\mathrm{N})$ and phosphorus $(\mathrm{P})$, exchangeable cations, texture, soil bulk density, water-pH and $\mathrm{KCl}-\mathrm{pH}$, and available-P in samples from natural (NS) and cultivated savannas (CS). Statistical analysis of the data included: a two way ANOVA, PCA and path analysis for the establishment of multicausal statistical models. The CS showed significant changes in the fertility associated with higher carbon sequestration in the order of $2,970 \mathrm{~kg} \mathrm{ha}^{-1}$ and a $50 \%$ increase in the electronegativity of the net coloidal charge density which is related with the LF SOM, COS and soil bulk density in comparion to the NS where variation factors were related to inorganic nature.

Key words: savannahs, carbon sink, isoelectric point, statistical models, sustainable agriculture.

\section{INTRODUCCION}

Los pastizales, que incluyen ecosistemas de sabanas, praderas, matorrales y agroecosistemas sembrados con pasto-forraje, cubren aproximadamente 3.500 millones de hectáreas, aproximadamente el $40 \%$ de la superficie terrestre y $70 \%$ del área agrícola global, contienen alrededor del $20 \%$ de las reservas mundiales de COS (10). La importancia de las sabanas y los pastizales, ademas de las grandes extensiones que ocupan, representa $30 \%$ de la producción primaria de toda la vegetación terrestre (13) por lo que el cambio de uso de estos ecosistemas supone un impacto sobre las tasas de acumulación o pérdida de carbono $(21 ; 34)$.

El COS almacena un estimado de 1.500 $\mathrm{PgC}$ en el primer metro de profundidad de los suelos, que supera el contenido combinado del carbono en la atmósfera, aproximadamente $800 \mathrm{PgC}$ y la vegetación terrestre, alrededor de $500 \mathrm{PgC}$ (10). En consecuencia todos los procesos asociados a la degradación de los suelos suponen importantes alteraciones del ciclo del carbono. La mayoría de los suelos en todo el mundo están lejos de sus umbrales de saturación de $\mathrm{C}$, por lo que representan un gran potencial para aumentar los inventarios de carbono, bien mediante la protección de las reservas existentes, así como maximizando el secuestro de carbono en los suelos (16). Los drásticos cambios de uso de los suelos en la zona intertropical y los acelerados procesos de su degradación, demuestran la clara visión, que de la vida de los suelos dependen la misma vida humana y las civilizaciones $(10 ; 42)$.

La erosión de los suelos se está acelerando en todos los continentes y está degradando unos 2.000 millones de hectáreas, lo que representa entre la quinta y la tercera parte de las tierras de cultivo de todo el mundo. Cada año la erosión y otras formas de degradación provocan pérdidas de entre 5 y 7 millones de hectáreas de suelos cultivables. La creciente necesidad de alimentos y leña, en los paises más pobres, han ocasionado una mayor deforestación e incremento de los cultivos en laderas con pronunciadas pendientes, que han inducido procesos muy acelerados de erosión. En el caso particular de América Latina y El Caribe más de 300 millones de hectáreas de suelos han sido afectadas por degradación. El Programa de Protección Ambiental de las Naciones Unidas, registró en esta región, un 16\% de los suelos degradados de todo el planeta, cuyas 
causas incluyeron: talas indiscriminadas, sobrepastoreo, expansión de la frontera de las áreas de agricultura, fuego y deforestación, (40) esta última, causa alrededor del $25 \%$ de la pérdida total de COS en todo el mundo (10).

En los bosques tropicales húmedos, el $60 \%$ del C se encuentra en la vegetación y un $40 \%$ en los suelos (10). En consecuencia, la deforestación y el fomento agrícola en los ecosistemas boscosos del Amazonas venezolano, equivale a un alto impacto sobre el ciclo del C, debido a la pérdida de los mecanismos de conservación de los suelos, a periodos de barbechos muy cortos (2-3 años) que inducen: una sobre explotación de los suelos, acelerados procesos de erosión, lenta recuperación de los ecosistemas intervenidos y pérdida de la biodiversidad. Todo lo anterior, en detrimento de la fertilidad química y la actividad biológica de los agroecosistemas establecidos en los bosques tropicales lluviosos $(20 ; 22 ; 43)$.

Las sabanas al norte del estado Amazonas en Venezuela, presentan fuertes restricciones para el desarrollo agrícola capitalista intensivo, situación que es común en las sabanas de Colombia (19) y Cerrados de Suramérica, pero que se ve acentuada en esta región, como consecuencia de la predominancia de suelos ácidos-arenosos, altamente meteorizados, de baja fertilidad química natural y drenaje excesivamente rápido, que favorece, el lavado de las bases intercambiables y una escasa retención del agua disponible en estos suelos $(4 ; 36)$. Por el contrario, estos ecosistemas pueden manejarse exitosamente mediante sistemas agroecológicos intensivos que acumulan carbono orgánico en los suelos, como el Pastoreo Racional Voisin, que no tributa al capital agroindustrial internacional, porque se sustenta en mecanismos que estimulan la biocenosis de los suelos que activan la biofertilidad natural, mediante diversas reacciones bioquímicas y numerosos procesos biológicos, que ocurren a partir de cambios cualitativos y cuantitativos sucesivos de la materia orgánica del suelo $(25 ; 27 ; 31)$.

La región en estudio presenta las precipitaciones en sabanas, más abundante en Venezuela y bajo estas condiciones los suelos ácidos estan frecuentemente asociados a constituyentes muy evolucionados, de baja a muy baja fertilidad natural debido a las fuertes pérdidas de arcilla, materia orgánica y bases intercambiables (23). En estos suelos predominan los sesquióxidos de hierro y aluminio y otros minerales secundarios con cargas dependientes del $\mathrm{pH}(4 ; 12)$. Cuando estos solidos entran en contacto con la solución acuosa, su superficie hidroxilada puede adsorber o liberar protones e hidroxilos de la solución. Esta adsorción está gobernada por la concentración de $\mathrm{H}^{+}$y de $\mathrm{OH}^{-}$, es decir por el pH. En medio ácido, la solución tiene un exceso de protones que podrían ser adsorbidos. Los grupos funcionales de la materia orgánica son igualmente capaces de tomar o liberar protones de la solución, porque su carga superficial depende del $\mathrm{pH}$ y de la concentración de la solución con la que está en contacto (44). En consecuencia, un manejo agronómico que permita inducir in situ incrementos perdurables del COS y que además estimule la actividad biológica, el metabolismo del suelo y retenga mayor humedad, es factor clave para el manejo agroecológico intensivo de los suelos ácidos-arenosos de estas sabanas.

Los objetivos de este trabajo fueron: evaluar el secuestro del COS en un Paleustults de sabana, cuatro años después del fomento agronómico de pasturas adaptadas a suelos ácidos, fertilizadas con fosfato de roca y NPK, como fuente complementaria; reco- 
nocer mediante un análisis estadístico de rutas, para sistemas multicausales, los factores que indujeron los mayores cambios en la densidad de carga neta coloidal (DCNC) en los tratamientos, sabana natural (SN) y sabana cultivada (SC) de acuerdo al peso estadístico de las variables orgánicas e inorgánicas; y determinar las bondades de estos agroecosistemas, como opción alterna al acelerado cambio de uso del bosque tropical lluvioso y sus riesgosas consecuencias ecológicas y ambientales.

\section{MATERIALES Y MÉTODOS}

El trabajo se realizó en una sabana de Trachypogon a $80 \mathrm{~m}$. s. n. m., localizada a $5^{\circ} 47^{\prime} \mathrm{N}$ y $67^{\circ} 30^{\prime} \mathrm{W}$, próxima a una comunidad indígena Guahiba, en Sabaneta de Guayabal, municipio Atures, al sur de Puerto Ayacucho, capital del estado Amazonas en Venezuela.

El clima del área de estudio es tropical de sabana con un período lluvioso que se inicia en el mes de abril y finaliza en diciembre. Los promedios anuales de precipitación y temperatura registran valores de $2.200 \mathrm{~mm}$ y $27^{\circ} \mathrm{C}$, respectivamente.

El área de estudio está ubicada en una zona que fisiográficamente corresponde a una transición entre Los Llanos al noroeste y el sistema montañoso del Macizo Central Guayanés al este, con similares condiciones de clima, material parental, relieve, actividad biológica y tiempo geológico. Los suelos, con una pendiente aproximada de $1 \%$, se caracterizan por procesos de lavado y empobrecimiento superficial de arcilla que le confieren una textura gruesa y drenaje interno rápido (4).
El material parental predominante está representado por rocas ácidas de origen ígneo provenientes del batolito de Parguaza, recubierto en gran parte de aluviones y suelos residuales (36). El suelo se identificó como un Arenic Paleustults, caracterizado por $\mathrm{pH}$ ácido $(4,5-5,5)$, bajos contenidos de materia orgánica del suelo (MOS) y baja capacidad de intercambio catiónico efectiva. La fracción arcillosa de estos suelos es dominada por caolinita y significativos contenidos de gibsita y óxidos de hierro libre. Presentan textura franco arenosa, con una alta proporción de arena a lo largo del perfil y estructura granular débil, que le confiere una alta permeabilidad y lixiviación excesiva durante los meses de máximas precipitaciones y un fuerte secado durante el periodo menos lluvioso $(4 ; 12)$.

La vegetación es la típica de las sabanas de Trachypogon con árboles (32). El estrato herbáceo estuvo representado principalmente por las gramíneas Trachypogon sp. y Axonopus sp.; mientras que el componente arbóreo por Curatella americana, Byrsonimia crassifolia y Bowdichia virgilioides.

\section{Selección del área experimental}

Para el diseño experimental se tomó un área de 360 m x 90 m dividida en dos partes iguales. De éstas, una se dejó como tratamiento de sabana natural (SN) de Trachypogon y, la otra fue cultivada (SC) con Urochloa dictyoneura en asociación con las leguminosas Stylosanthes capitata y Centrosema macrocarpum; la SC fue sometida a dos pases de rastra y una fertilización al voleo, con dosis de 1,0 y $0,3 \mathrm{Mg} \mathrm{ha}^{-1}$ de roca fosfórica (RF) y NPK (12-24-12), respectivamente. Este tratamiento también incluyó un pastoreo continuo con ovejos (Ovis aries) con una capacidad de carga de animal equivalente a 1,2 $\mathrm{UA} \mathrm{ha}^{-1}$

Revista FAVE - Ciencias Agrarias 18 (2) 2019 
Cuatro años después, los tratamientos SN y SC fueron muestreados al azar en 12 puntos, a razón de $0,01 \mathrm{~m}^{2}(0,1 \mathrm{~m} \times 0,1 \mathrm{~m})$ para las profundidades de 0-3,0; 3,1-6,0 y $6,1-10,0 \mathrm{~cm}$. En cada tratamiento se obtuvieron tres muestras compuestas, al mezclar cuatro unidades simples de muestreo que tenían en común: el tratamiento, la profundidad y el tamizado. Estas muestras compuestas de suelos, estadísticamente independientes entre sí, se secaron a temperatura ambiente y luego fueron tamizadas, homogeneizadas y cuarteadas para los análisis físicos y químicos.

\section{Análisis de suelo}

Los análisis incluyeron tres réplicas de laboratorio de cada una de las muestras compuestas. Para determinar el COS y la fracción ligera de la materia orgánica del suelo, (FL MOS) se empleó el método de ignición (1) y el método por flotación en agua (2). Los contenidos totales de $\mathrm{N}$ y $\mathrm{P}$, se obtuvieron, primero, por el método de digestión con $\mathrm{H}_{2} \mathrm{SO}_{4}$ y $\mathrm{H}_{2} \mathrm{O}_{2}$ (1) y posteriormente, oxidación húmeda (método Kjeldhal) para $\mathrm{N}$ (1) y formación del complejo azul de fosfato-molibdato para P (24). Para el fósforo disponible (P-disponible) se utilizó el método de Olsen (28). La textura y la densidad aparente ( $\mathrm{Da}$ ) se determinó por Bouyoucos y el método del hoyo en campo (5), respectivamente. El pH se determinó mediante un electrodo de vidrio sumergido en suspensión suelo:solución (agua o $\mathrm{KCl} 1 \mathrm{~N}) 1: 5$; y la densidad de carga neta coloidal (DCNC) del suelo se calculó por diferencia entre estos valores de $\mathrm{pH}(\Delta \mathrm{pH}=$ $\mathrm{pH}_{\mathrm{KCl}}-\mathrm{pH}_{\text {agua }}$ ) para determinar los cambios electroquímicos del suelo $(37 ; 39)$. La determinación de las bases intercambiables: $\mathrm{Ca}^{+2}, \mathrm{Mg}^{+2}, \mathrm{Na}^{+}$y K $\mathrm{K}^{+}$(datos no mostrados), fue realizada por espectrofotometría de $\mathrm{ab}$ - sorción atómica empleando una solución extractora de acetato de amonio $1 \mathrm{M}$ a $\mathrm{pH}$ 7 (2).

\section{Análisis estadístico}

Se realizó un análisis de varianza de dos vías para conocer la significación estadística de las diferencias entre los tratamientos SN y SC y entre las profundidades de cada muestra de suelo. Se aplicó una prueba $a$ posteriori de diferencias mínimas significativas al $95 \%$ de confianza. Un análisis de las propiedades composicionales de suelo, previo al establecimiento de las pasturas, en los vértices de cuadrículas de $10 \mathrm{~m}$ x 10 $\mathrm{m}$ demarcadas sobre la superficie experimental, mostró la homogeneidad del suelo y permitió atribuir las diferencias encontradas al efecto de los tratamientos. La Tabla 1 muestra las propiedades seleccionadas de un estudio previo de los suelos en los primeros $10 \mathrm{~cm}$ del epipedón, que confirmó la homogeneidad de los suelos a esta profundidad en el área de estudio (15). Se realizó un análisis de componentes principales (ACP) sobre una matriz de correlación estandarizada, que permitió homogenizar la varianza de cada variable a 1 . Finalmente un análisis de rutas para sistemas multicausales, permitió establecer los modelos estadísticos de acuerdo a los factores que indujeron los mayores cambios en la DCNC en los tratamientos SN y SC. El análisis de rutas es una técnica estadística de exploración y modelación que permite describir y establecer hipótesis de funcionamiento de un sistema y crear un esquema teórico-matemático sobre su funcionamiento (29). Los resultados se presentan en esquemas donde los valores de los coeficientes de regresión parcial se visualizan sobre las flechas rectas, los coeficientes de correlación simple sobre las flechas curvas, $\mathrm{R}^{2}$ corresponde al 
coeficiente de determinación de la regresión múltiple y $\mathrm{U}$ representa las variables que no fueron medidas y en consecuencia la varianza de los datos no explicada por el modelo en ese punto. Los análisis estadísticos de rutas incluye todo el conjunto de estos datos.

\section{RESULTADOS}

La Tabla 2 presenta los porcentajes de arena, limo, arcilla y el valor de la densidad aparente de los suelos en los tratamientos: sabana cultivada (SC) y sabana natural (SN). Ambos tratamientos presentaron contenidos promedios de arena de alrededor del $90 \%(88,8-93,3)$ y porcentajes muy similares de arcilla $(2,7-4,0)$ a las distintas profundidades. Los principales cambios ocurrieron en la fracción limo del tratamiento $\mathrm{SC}$ a las profundidades de 3,1-6,0 y $6,1-10,0 \mathrm{~cm}$ donde hubo un incremento de alrededor del doble en comparación con la $\mathrm{SN}$; y en la Da que registró un incremento estadísticamente significativo en las muestras provenientes de la capa más profunda (3,1-6,0 y $6,1-10,0 \mathrm{~cm})$ al compararlo con los valores promedio de Da de las capas de los suelos más superficiales (Tabla 2).

La Tabla 3, reúne los resultados de $\mathrm{pH}_{\text {agua }}, \mathrm{pH}_{\mathrm{KCl}}$ y la DCNC en cada uno de los tratamientos. Los valores de $\mathrm{pH}_{\mathrm{KCl}}$ de la SC, mostraron diferencias estadísticamente significativas con el resto de los tratamientos, al igual que la DCNC, correspondiéndole a la SC un carácter $50 \%$ más electronegativo en comparación con la SN. Estas diferencias representaron incrementos de 0,5 y 0,6 unidades de $\mathrm{pH}$, estadísticamente significativas.

Los contenidos de carbono orgánico del suelo (COS) y fracción ligera de la materia orgánica del suelo (FL MOS), para las tres profundidades y ambos tratamientos se presentan en la Tabla 4. El COS en la SN disminuyó con la profundidad particularmente en la capa más profunda $(6,1-10,0 \mathrm{~cm})$. La $\mathrm{SC}$ presentó el mayor contenido de COS y mostró diferencias significativas a las profundidades de 3,1-6,0 y $6,1-10,0 \mathrm{~cm}$ en comparación con la SN. La FL MOS de la $\mathrm{SC}$ mostró diferencias significativas a todas las profundidades, duplicándose los valores en comparación con la SN. Los valores más altos se obtuvieron en las capas más superficiales de la SC.

Tabla 1. Propiedades seleccionadas de los suelos $(0-10 \mathrm{~cm})$ en los tratamientos SN y SC.

\begin{tabular}{|c|c|c|c|c|c|c|c|}
\hline \multirow{3}{*}{ Tratamientos } & \multirow{2}{*}{$\mathrm{Da}$} & \multirow{2}{*}{ RMA } & \multirow{2}{*}{ Arena } & \multirow{2}{*}{ MOS } & \multicolumn{3}{|c|}{$P$} \\
\hline & & & & & disponible & orgánico & total \\
\hline & $\mathrm{g} \mathrm{cm}^{-3}$ & & $\%$ & & & $-\mu \mathrm{g} / \mathrm{g}$ & \\
\hline SN & $1,50 \mathrm{a}$ & $8,61 \mathrm{a}$ & $94,0 \mathrm{a}$ & $1,07 \mathrm{a}$ & $4,8 \mathrm{a}$ & $42,4 a$ & $149,3 a$ \\
\hline SC & $1,48 a$ & $8,84 a$ & $92,8 a$ & $0,74 a$ & $6,2 \mathrm{a}$ & $42,5 \mathrm{a}$ & $134,2 \mathrm{a}$ \\
\hline
\end{tabular}

Nota: RMA, retención máxima de agua. Letras diferentes significan medias diferentes (U. Mann-Whitnney,

$P<0,05)$. Las comparaciones se realizaron para una misma variable entre el sistema de manejo y su control

(15). 
Tabla 2. Propiedades físicas del suelo en los tratamientos sabana natural (SN) y sabana cultivada (SC) a distintas profundidades del suelo.

\begin{tabular}{|c|c|c|c|c|c|c|c|c|}
\hline \multirow{3}{*}{$\begin{array}{l}\text { Prof. } \\
\mathrm{cm}\end{array}$} & \multicolumn{2}{|c|}{ Arena } & \multicolumn{2}{|c|}{ Limo } & \multicolumn{2}{|c|}{ arcilla } & \multicolumn{2}{|c|}{$\mathrm{Da}$} \\
\hline & \multicolumn{6}{|c|}{$\%$} & \multicolumn{2}{|c|}{$\mathrm{g} \mathrm{cm}^{-3}$} \\
\hline & SC & SN & SC & SN & SC & SN & SC & SN \\
\hline \multirow[t]{2}{*}{$0,0-3,0$} & 90,5 & 92,0 & 6,2 & 4,7 & 3,3 & 3,3 & $1,4 \mathrm{Ab}$ & $1,4 \mathrm{Ac}$ \\
\hline & $\pm 1,10$ & $\pm 0,00$ & $\pm 1,22$ & $\pm 1,15$ & $\pm 1,15$ & $\pm 1,15$ & $\pm 0,1$ & $\pm 0,2$ \\
\hline \multirow[t]{2}{*}{$3,1-6,0$} & 88,8 & 92,7 & 7,2 & 3,3 & 4,0 & 4,0 & $1,5 \mathrm{Bb}$ & $1,8 \mathrm{Ab}$ \\
\hline & $\pm 1,06$ & $\pm 1,15$ & $\pm 2,08$ & $\pm 1,15$ & $\pm 2,00$ & $\pm 2,00$ & $\pm 0,1$ & $\pm 0,3$ \\
\hline \multirow[t]{2}{*}{$6,1-10,0$} & 89,8 & 93,3 & 7,5 & 4,0 & 2,7 & 2,7 & 1,9 Ba & $2,2 \mathrm{Aa}$ \\
\hline & $\pm 1,15$ & $\pm 1,15$ & $\pm 1,15$ & $\pm 0,00$ & $\pm 1,15$ & $\pm 1,15$ & $\pm 0,1$ & $\pm 0,3$ \\
\hline
\end{tabular}

Nota: Letras distintas denotan diferencias estadística significativas ( $p<0,05 ; N=3$ ). Los valores precedidos de los simbolos \pm indican la desviación estándar de la media. La primera letra mayúscula denota las diferencias entre los tratamientos, SC y SN, y la segunda minúscula, las diferencias por efecto de la profundidad.

Tabla 3. Cambios en el pH y la densidad de carga neta coloidal (DCNC) en los tratamientos sabana cultivada (SC) y sabana natural (SN) según la profundidad del suelo.

\begin{tabular}{|c|l|l|l|l|l|l|}
\hline \multirow{2}{*}{$\begin{array}{c}\text { Prof } \\
(\mathrm{cm})\end{array}$} & \multicolumn{2}{|c|}{$\mathrm{pH}_{\text {agua }}$} & \multicolumn{2}{c|}{$\mathrm{pH}_{\mathrm{KCl}}$} & \multicolumn{2}{c|}{$\mathrm{DCNC}$} \\
\cline { 2 - 7 } & \multicolumn{1}{|c}{$\mathrm{SC}$} & $\mathrm{SN}$ & \multicolumn{1}{c|}{$\mathrm{SC}$} & $\mathrm{SN}$ & \multicolumn{1}{c|}{$\mathrm{SC}$} & \multicolumn{1}{c|}{$\mathrm{SN}$} \\
\hline \multirow{3}{*}{$0,0-3,0$} & 5,4 & 5,4 & 4,3 & 4,7 & $-1,2$ & $-0,7$ \\
& $\pm 0,01$ & $\pm 0,02$ & $\pm 0,03$ & $\pm 0,05$ & & \\
& $\mathrm{Aa}$ & $\mathrm{Aa}$ & $\mathrm{Ba}$ & $\mathrm{Aa}$ & $\mathrm{Ba}$ & $\mathrm{Ab}$ \\
\hline \multirow{3}{*}{$3,1-6,0$} & 5,3 & 5,3 & 4,2 & 4,8 & $-1,1$ & $-0,5$ \\
& $\pm 0,11$ & $\pm 0,07$ & $\pm 0,05$ & $\pm 0,04$ & & \\
& $\mathrm{Aa}$ & $\mathrm{Aa}$ & $\mathrm{Ba}$ & $\mathrm{Aa}$ & $\mathrm{Ba}$ & $\mathrm{Aa}$ \\
\hline \multirow{3}{*}{$6,1-10,0$} & 5,3 & 5,2 & 4,3 & 4,8 & $-1,0$ & $-0,6$ \\
& $\pm 0,04$ & $\pm 0,05$ & $\pm 0,02$ & $\pm 0,05$ & & \\
& $\mathrm{Aa}$ & $\mathrm{Aa}$ & $\mathrm{Ba}$ & $\mathrm{Aa}$ & $\mathrm{Ba}$ & $\mathrm{Aa}$ \\
\hline
\end{tabular}

Nota: Letras distintas denotan diferencias estadística significativas ( $p<0,05 ; N=3)$. Los valores precedidos de los simbolos \pm indican la desviación estándar de la media. La primera letra mayúscula denota las diferencias entre los tratamientos, SC y SN, y la segunda minúscula, las diferencias por efecto de la profundidad. 


\section{A. D. Ojeda-Falcón et al.}

El contenido de $\mathrm{N}$ en general, tiende a disminuir con la profundidad, así la SC presentó el valor más bajo $\left(206 \mathrm{mg} \mathrm{kg}^{-1}\right)$ en la capa más profunda y resultó estadísticamente distinto al compararlo con el contenido de $\mathrm{N}$ de las capas superiores del suelo (0-3,0 y 3,1-6,0 cm) las cuales no mostraron diferencias entre sí. Los niveles de $\mathrm{N}$ en ambos tratamientos no presentaron diferencias significativas, excepto para la capa más superficial del suelo (Tabla 4). En el caso del P, se encontró un incremento significativo en la $\mathrm{SC}$, tanto de $\mathrm{P}$ total como de $\mathrm{P}$ disponible a las tres profundidades respecto a la $\mathrm{SN}$. No se observó un patrón claro de acumulación de $\mathrm{P}$ con la profundidad en ninguno de los tratamientos (Tabla 4).

La relación $\mathrm{C} / \mathrm{P}$ de la $\mathrm{SN}$ en las primeras dos profundidades duplicó el valor obtenido para la SC, lo cual no es de extrañar debido al incremento del contenido de P en la sabana fertilizada con RF. Para las mismas profundidades, la relación $\mathrm{C} / \mathrm{N}$ mostró igualmente un ligero aumento en la $\mathrm{SN}$ respecto a la $\mathrm{SC}$, pero con una diferencia mucho menor porque los incrementos en $\mathrm{N}$, por efecto de la fertilización química, no se observaron después de los cuatro años de cultivo. El patrón anteriormente descrito para la relación $\mathrm{C} / \mathrm{N}$ se modificó a la profundidad de 6,1-10,0 cm, mientras que a esta misma profundidad, la relación $\mathrm{C} / \mathrm{P}$ sólo mostró una pequeña diferencia entre ambos tratamientos (Tabla 4).

La Tabla 5 presenta el balance del COS ( $\mathrm{kg} \mathrm{ha}^{-1}$ ) acumulado originalmente en la SN y en la SC cuatro años después de iniciado el fomento agronómico de las pasturas, para las tres profundidades analizadas. El balance total del COS arrojó un incremento de 2.970 $\mathrm{kg} \mathrm{ha}^{-1}$ en los primeros $10 \mathrm{~cm}$ de este suelo.

Tabla 4. Propiedades químicas seleccionadas de los suelos en la SC y la SN

\begin{tabular}{|c|c|c|c|c|c|c|c|c|c|c|c|c|c|c|}
\hline \multirow{2}{*}{$\begin{array}{l}\text { Prof } \\
\mathrm{cm}\end{array}$} & & & \multirow{2}{*}{\multicolumn{2}{|c|}{$\begin{array}{c}\text { FL MOS } \\
\%\end{array}$}} & \multicolumn{2}{|c|}{$\mathrm{N}$ total } & \multicolumn{2}{|c|}{$\mathrm{P}$ total } & \multicolumn{2}{|c|}{ P-disponible } & \multirow{2}{*}{\multicolumn{2}{|c|}{$\mathrm{C} / \mathrm{N}$}} & \multirow{2}{*}{\multicolumn{2}{|c|}{$\mathrm{C} / \mathrm{P}$}} \\
\hline & \multicolumn{2}{|c|}{$\mathrm{g} \mathrm{kg}^{-1}$} & & & \multicolumn{6}{|c|}{$\mathrm{mg} \mathrm{kg}^{-1}$} & & & & \\
\hline & $\mathrm{SC}$ & SN & $\mathrm{SC}$ & SN & $\mathrm{SC}$ & SN & SC & SN & $\mathrm{SC}$ & SN & SC & SN & $\mathrm{SC}$ & $\mathrm{SN}$ \\
\hline \multirow{3}{*}{$0,0-3,0$} & 6,8 & 6,7 & 0,5 & 0,2 & 279 & 210 & 122 & 62,0 & 9,5 & 3,0 & & & & \\
\hline & $\pm 1,3$ & $\pm 0,4$ & $\pm 0,06$ & $\pm 0,9$ & $\pm 23,6$ & $\pm 25,6$ & $\pm 4,0$ & $\pm 0,4$ & $\pm 3,5$ & $\pm 0,0$ & 24,4 & 31,8 & 55,6 & 108 \\
\hline & $\mathrm{Aa}$ & $\mathrm{Aa}$ & $\mathrm{Aa}$ & $\mathrm{Ba}$ & $\mathrm{Aa}$ & $\mathrm{Ba}$ & $A b$ & $\mathrm{Bab}$ & $\mathrm{Aa}$ & $\mathrm{Ba}$ & & & & \\
\hline \multirow{3}{*}{$3,1-6,0$} & 7,1 & 6,2 & 0,3 & 0,15 & 254 & 217 & 147 & 66,0 & 9,0 & 1,5 & & & & \\
\hline & $\pm 0,8$ & $\pm 0,9$ & $\pm 0,06$ & $\pm 0,3$ & $\pm 19,1$ & $\pm 40,7$ & $\pm 12,6$ & $\pm 3,3$ & $\pm 1,4$ & $\pm 0,7$ & 27,9 & 28,5 & 48,2 & 93,9 \\
\hline & $\mathrm{Aa}$ & $\mathrm{Ba}$ & $A b$ & Bab & $\mathrm{Aa}$ & $\mathrm{Aa}$ & $\mathrm{Aa}$ & $\mathrm{Ba}$ & $\mathrm{Aa}$ & $\mathrm{Ba}$ & & & & \\
\hline \multirow{3}{*}{$6,1-10,0$} & 7,2 & 4,7 & 0,2 & 0,11 & 206 & 191 & 95 & 57,5 & 7,5 & 2,0 & & & & \\
\hline & $\pm 0,5$ & $\pm 1,5$ & $\pm 0,05$ & $\pm 0,1$ & $\pm 57,5$ & $\pm 41,7$ & $\pm 4,2$ & $\pm 5,6$ & $\pm 0,7$ & $\pm 1,4$ & 34,9 & 24,6 & 75,9 & 81,7 \\
\hline & $\mathrm{Aa}$ & $\mathrm{Bb}$ & $A c$ & $\mathrm{Bb}$ & $A b$ & $\mathrm{Aa}$ & $A c$ & $\mathrm{Bb}$ & $\mathrm{Aa}$ & $\mathrm{Ba}$ & & & & \\
\hline
\end{tabular}

Nota: Letras distintas denotan diferencias estadística significativas $(p<0,05 ; N=6)$. Los valores precedidos de los simbolos \pm indican la desviación estándar de la media. La primera letra mayúscula denota las diferencias entre los tratamientos, SC y SN, y la segunda minuscula, las diferencias por efecto de la profundidad

Tabla 5. Balance del COS $\left(\mathrm{kg} \mathrm{ha}^{-1}\right)$ en los suelos de los tratamientos SC y SN.

\begin{tabular}{|c|r|r|r|}
\hline \multirow{2}{*}{$\begin{array}{c}\text { Prof. } \\
\text { cm }\end{array}$} & \multicolumn{2}{|c|}{ COS } & \multirow{2}{*}{$\Sigma$ SOS $=$ SC-SN } \\
\cline { 2 - 3 } & SC & SN & \\
\hline $0,0-3,0$ & 9.520 & 9.380 & 140 \\
\hline $3,1-6,0$ & 10.650 & 11.160 & -510 \\
\hline $6,1-10,0$ & 13.680 & 10.340 & 3.340 \\
\hline$\Sigma=0-10$ & 33.850 & 30.880 & 2.970 \\
\hline
\end{tabular}


La Figura 1 muestra el Análisis de Componentes Principales (ACP) que permitió reconocer las variables que más contribuyeron a explicar $72,5 \%$ de la varianza en los dos primeros componentes que separó los tratamientos y las distintas profundidades (Tabla 6). La Tabla 7 muestra los autovectores, el peso de las variables con mayor explicación de la varianza y los valores de $\mathrm{r}$ $\mathrm{y} \mathrm{r}^{2}$. En el primer componente las variables que presentaron mayor explicación fueron: $\mathrm{pH}_{\mathrm{KCl}}(0,89)$, FL MOS $(-0,88), \mathrm{P}(-0,87)$, y DCNC $(-0,95)$. En consecuencia los suelos de la SN fueron separados principalmente por el $\mathrm{pH}_{\mathrm{KC} C}$, en las capas más profundas, mientras que la separación de los suelos de la SC, fue atribuida a la FL MOS, P, y la DCNC. En el segundo componente las variables que presentaron mayor explicación fueron: $\mathrm{Da}(0,63){\text { y } \mathrm{pH}_{\text {agua }}}(-0,68)$, lo que indujo la separación de los suelos de la SC atribuido principalmente al limo y la Da en las profundidades: $3,1-6,0$ y $6,1-10,0 \mathrm{~cm}$; mientras que el $\mathrm{pH}_{\text {ava }}$ y el $\mathrm{pH}_{\mathrm{KC}}$ separó ambos tratamientos a la profundidad de $0-3,0$ $\mathrm{cm}$.

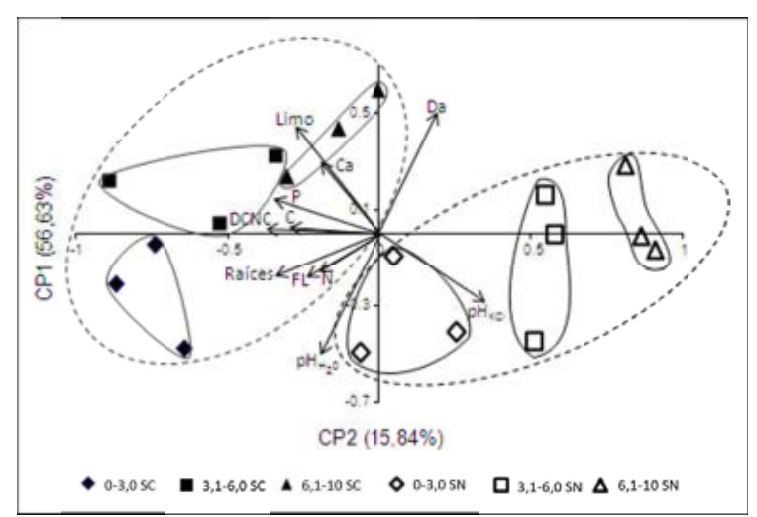

Figura 1. Análisis de Componentes Principales para los tratamientos sabana natural y sabana cultivada a distintas profundidades del suelo.

Tabla 6. Autovalores y porcentajes de la varianza explicada y acumulada de los componentes principales.

\begin{tabular}{|l|r|r|r|r|r|}
\hline \multirow{2}{*}{} & \multicolumn{5}{|c|}{ Componente número } \\
\cline { 2 - 6 } & \multicolumn{1}{|c|}{1} & \multicolumn{1}{c|}{2} & \multicolumn{1}{c|}{3} & \multicolumn{1}{c|}{4} & \multicolumn{1}{c|}{5} \\
\hline Autovalores & 6,23 & 1,74 & 1,05 & 0,63 & 0,51 \\
\hline Porcentaje de la varianza & 56,63 & 15,85 & 9,52 & 5,76 & 4,70 \\
\hline Porcentaje acumulado & 56,63 & 72,48 & 82,00 & 87,76 & 92,46 \\
\hline
\end{tabular}


Tabla 7. Autovectores de la matriz de componentes principales (CP).

\begin{tabular}{|l|r|r|r|r|}
\hline Variables & $\mathrm{CP} 1$ & $\mathrm{CP} 2$ & $\mathrm{r}$ & $\mathrm{r}^{2}$ \\
\hline $\mathrm{PH}_{\mathrm{KCl}}$ & 0,359 & $-0,28$ & 0,89 & 0,80 \\
\hline $\mathrm{FL} \mathrm{MOS}$ & $-0,353$ & $-0,12$ & $-0,88$ & 0,78 \\
\hline $\mathrm{P}$ & $-0,347$ & 0,12 & $-0,87$ & 0,75 \\
\hline $\mathrm{DCNC}$ & $-0,381$ & 0,04 & $-0,95$ & 0,90 \\
\hline $\mathrm{Da}$ & 0,478 & 0,23 & 0,63 & 0,39 \\
\hline $\mathrm{pH}_{\text {agua }}$ & $-0,516$ & $-0,27$ & $-0,68$ & 0,46 \\
\hline
\end{tabular}

Los modelos teóricos-matemáticos obtenidos por análisis de ruta, paso a paso, para los tratamientos SN y SC, se muestran en las Figs. 2 y 3. La variabilidad explicada de los modelos teóricos-matemáticos y los $\mathrm{R}^{2}$ en los tratamiento $\mathrm{SN}$ y $\mathrm{SC}$, fueron muy altos $(0,99)$, al identificar las fuentes de variabilidad de DCNC (Fig. 2). Valores más bajos de $\mathrm{R}^{2}, 0,67$ y 0,76 , los presentó el tratamiento SC, al explicar las fuentes de variabilidad del $\mathrm{pH}_{\text {agua }}$ y el $\mathrm{pH}_{\mathrm{KCl}}$, respectivamente (Fig. 3).

Las fuentes de variación de la DCNC en el tratamientos $\mathrm{SN}$, provino principalmente de factores de naturaleza inorganica, (Da, $\mathrm{P}$, limo y $\mathrm{Ca}$ intercambiable) en contraste con el tratamiento SC, cuyas fuentes de variación fueron principalmente de naturaleza orgánicas (FL MOS, COS) o relacionados con la Da, en este caso particular debido a los ya comentados incrementos de la MOS. La Fig. 3 muestra para el tratamiento SC una intensidad muy fuerte $(0,82)$ asociada a la FL MOS como fuente de variación del $\mathrm{pH}_{\text {agua }}$, que constituye la variable independiente con mayor influencia directa $(1,1)$ sobre la DCNC como variable dependiente, seguido del $\mathrm{pH}_{\mathrm{KCl}}(0,41) \mathrm{y}$, el $\mathrm{P}$ y $\mathrm{N}$, que mostraron muy bajas intensidades directas sobre la DCNC (Fig. 2). Por contraste, en la $\mathrm{SN}$ la variable independiente con mayor intensidad sobre la DCNC fue el $\mathrm{pH}_{\text {agua' }}$, mientras que el $\mathrm{pH}_{\mathrm{KCl}}$ y el Ca intercambiable, mostraron una relación de causalidad negativa sobre esta variable dependiente (Fig. 2).

En el tratamiento $\mathrm{SN}$ la variable independiente con mayor intensidad de causalidad negativa fue $\mathrm{Da}$, mientras que las variables $\mathrm{N}, \mathrm{P}$ y Ca intercambiable, presentaron intensidades de causalidad positivas sobre el $\mathrm{pH}_{\text {agua }}$ como variable dependiente que explicó $78 \%$ de la variabilidad del modelo (Fig. 3); mientras que las variables independientes $\mathrm{Da}$, limo y $\mathrm{Ca}$, ejercieron una relación de causalidad negativa sobre el $\mathrm{pH}_{\mathrm{KCl}}$ como variable dependiente, con un $\mathrm{R}^{2}=0,93$ y explicó $74 \%$ de la variabilidad de este modelo en el tratamiento de la SN. Por contraste, las variables independientes con mayor intensidad sobre el $\mathrm{pH}_{\mathrm{KCl}}$ en el tratamiento SC fueron: FL MOS, seguido por la Da y el COS (Fig. 3). 


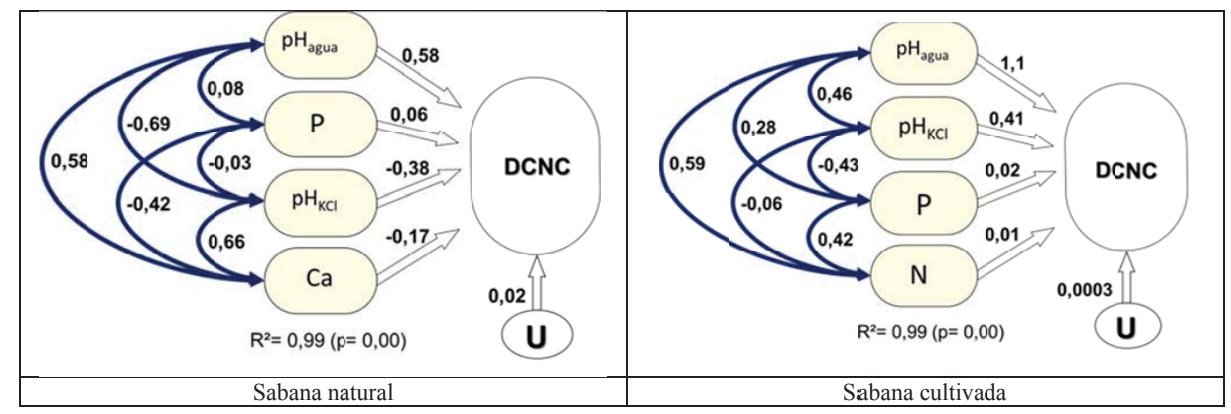

Figura 2. Modelos teóricos-matemáticos de los factores que afectan la densidad de carga neta coloidal (DCNC) como variable dependiente en los tratamientos sabana natural (a la izquierda) y sabana cultivada (a la derecha).

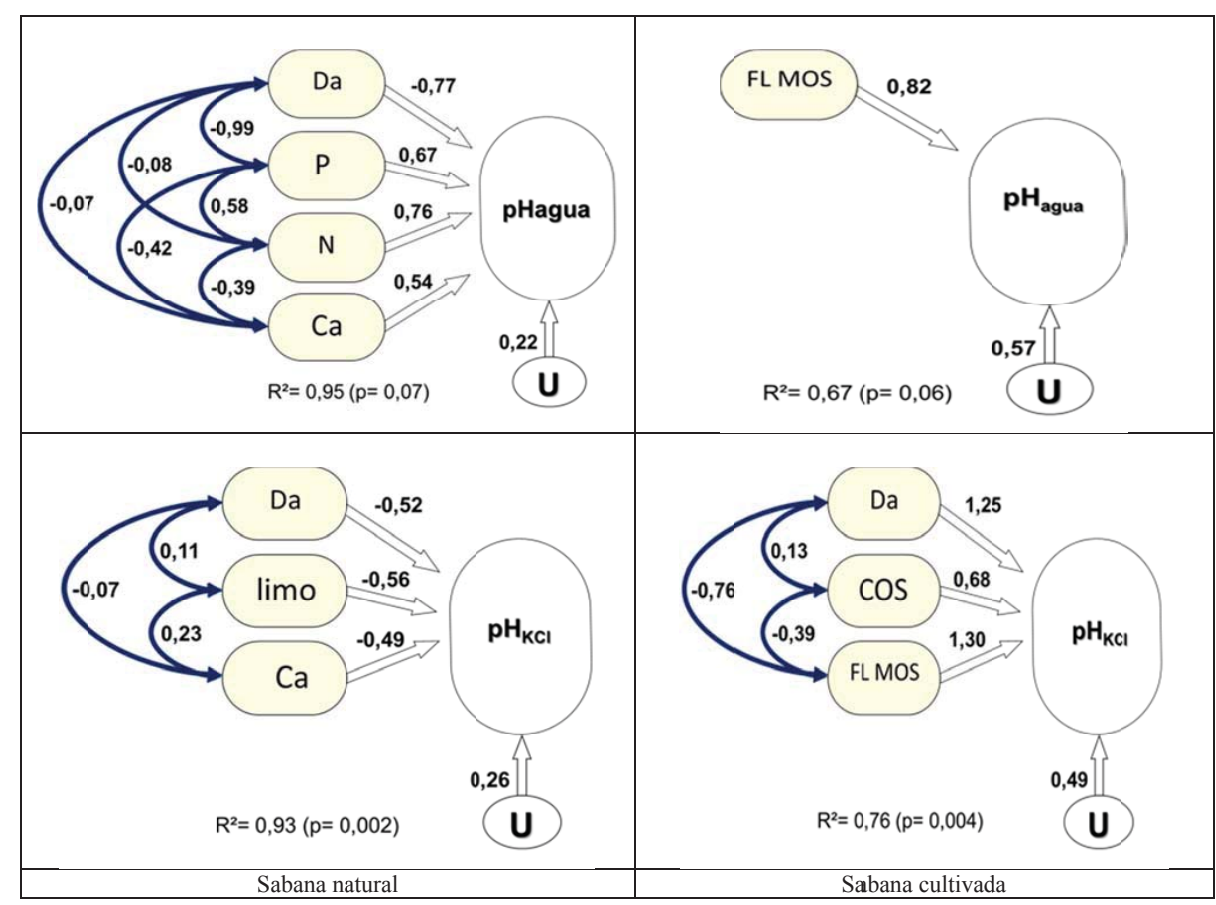

Figura 3. Modelos teóricos-matemáticos de los factores que afectan el pHagua y el $\mathrm{pH}_{\mathrm{KCl}} \mathrm{como}$ variables dependientes en los tratamientos sabana natural (a la izquierda) y sabana cultivada (a la derecha). 


\section{DISCUSIÓN}

El incremento en Da con la profundidad en ambos tratamientos, alcanzó valores superiores al límite considerado crítico (1,6 g $\mathrm{cm}^{-3}$ ) para suelos de textura arenosa, lo que sugiere una mayor compactación del suelo a partir de los $3 \mathrm{~cm}$ de profundidad, más acentuado en el caso de la SN. Los cambios en la fracción limo del suelo podrían estar asociados al efecto conjunto del arado del suelo de la SC durante la fertilización y siembra, pero sobretodo a una mayor actividad biológica de la comunidad de lombrices de tierra, que en mejores condiciones de retención de humedad y protección de la radiación solar, por la cobertura herbácea establecida, indujo mayor mezclado entre las capas del suelo, mayor permanencia de estos organismos en las capas superiores y mayor acarreo de la fracción limo en la SC en contraste con la SN.

Una observación similar realizaron en suelos con características parecidas, en las sabanas de Carimagua (Colombia) al encontrar diferencias significativas de la densidad aparente entre las estructuras biogénicas, provenientes de las lombrices: $M$. carimaguensis y Andiodrilus sp y agregados del suelo del mismo tamaño (7). Sin embargo, una mayor estabilidad de estas estructuras biogénicas, no pudo ser asociada a un mayor contenido de carbohidratos y en consecuencia los autores atribuyeron los cambios a mecanismos físicos que indujeron su endurecimiento, relacionado a un consumo selectivo por las lombrices de las fracciones limo y arcilla. Si bien las poblaciones de oligoquetos de las parcelas estudiadas no fue analizada, observaciones independientes realizadas para sistemas de sabanas similares, proxima al área de estudio, indicaron la presencia de una población endógena de lombrices de tierra que incrementó en biomasa y densidad en los sistemas orgánicos agroforestales (3). Otros autores enfatizan que la bioturbación del suelo juega un papel clave en el desarrollo de su profundidad, en su estructura y la translocación del C entre las capas del suelo (11).

Los cambios fisicoquímicos asociados al $\Delta \mathrm{pH}$ permitieron inferir la densidad de carga neta coloidal (DCNC) en estos suelos. Estos cambios son relevantes porque están directamente relacionados con una mayor capacidad de intercambio catiónico $(5 ; 23)$. El pH de este suelo al oscilar entre valores de 4,5 y 5,0, induce valores por debajo del punto de carga neta cero (PCNC), lo que determina una densidad de carga neta positiva y un predominio de los fenómenos de intercambio aniónico en la superficie de los principales coloides inorgánicos, característicos de los suelos ácidos altamente meteorizados, como los suelos Oxisoles y algunos Ultisoles, dominados por coloides de carga variable, en los cuales el valor del PCNC y el pH del suelo definen la capacidad de intercambio catiónico (44).

En consecuencia, los cambios de fertilidad observados aquí se asocian a fenómenos de naturaleza electroquímica, determinados por los valores de $\mathrm{pH}_{\text {agua }}$, que no mostraron diferencias entre los tratamientos, en contraste con valores significativamente más bajos de $\mathrm{pH}_{\mathrm{KCl}}$ en los suelos y las profundidades del tratamiento SC. Lo anterior tuvo influencia directa y correlativa sobre el incremento en la DCNC en las muestras de suelo de la SC, pero al parecer estos cambios no se pueden atribuir al fenómeno de solubilización de la roca fosfórica, pues los valores de $\mathrm{pH}_{\text {agua }}$ permanecieron prácticamente constantes, lo que nos induce a pensar que la carga generada provino básicamente de la materia orgánica acumulada 
durante el desarrollo de las pasturas. En estos suelos ácidos-arenosos, los cambios del COS son aún más relevantes debido al rol que tienen las cargas variables dependientes del $\mathrm{pH}$ de los coloides orgánicos y los valores de $\mathrm{pH}$ normalmente $(4,5-5,0)$ por encima de su PCNC alrededor de 3,0.

La tasa de acumulación de COS en este trabajo fue de $743 \mathrm{~kg} \mathrm{ha}^{-1}$ año $^{-1}$ en los primeros $10 \mathrm{~cm}$ de profundidad, donde ocurre la mayor influencia de la rizósfera de las pasturas. En las sabanas de Colombia y Venezuela se han registrado incrementos de $\mathrm{C}$ con valores entre 500 y $3.000 \mathrm{~kg} \mathrm{ha}^{-1}$ año ${ }^{-1}$ de $\mathrm{C}$ a una profundidad de $0-40 \mathrm{~cm}$ (6). Valores más modestos del orden de 140 $\mathrm{kg} \mathrm{ha}^{-1}$ año ${ }^{-1}$ de $\mathrm{C}$ han sido registrados para sabanas tropicales (13). En pasturas bien manejadas, el ingreso de materia orgánica proveniente de la bosta y del sistema radical, ha sido calculado en el orden de 14.467 $\mathrm{kg} \mathrm{ha}^{-1}$ año $^{-1}$ de $\mathrm{CO}_{2}$ (25) que equivalen en C a $3.946 \mathrm{~kg} \mathrm{ha}^{-1} \mathrm{año}^{-1}$ en ausencia de pérdidas hacia otros compartimientos del ecosistema (31).

Los efectos de los tratamientos en este trabajo, mostraron importantes cambios de fertilidad (23), que coincide con los obtenidos en sabanas de Colombia cultivadas con Brachyaria dictyoneura, al determinar un año después del fomento agronómico, que el $50 \%$ del volumen de raíces totales $(2,1$ $\mathrm{Mg} \mathrm{ha}^{-1}$ ) provino de los primeros $10 \mathrm{~cm}$ del suelo (33). Un aumento de $1 \mathrm{t}$ del COS (cifra muy próxima a la tasa de acumulación anual de COS obtenida en este trabajo) en tierras degradadas, se relacionó con el aumento de los rendimientos, por ejemplo, de maíz entre $10-20 \mathrm{~kg} \mathrm{ha}^{-1}$, lo que confirma que el manejo sostenible del suelo, al aumentar las reservas del COS, debe adoptarse como sistema alimentario más sostenible
(17) en lugar de la agricultura industrial de altos insumos y profundo impacto ambiental sobre los bienes comunes de suelos y aguas.

La información aquí obtenida puede abrirle perspectivas al manejo intensivo agroecológico de los suelos con vocación silvopastoril y/o agroforestal, como estrategia para incrementar la acumulación del COS, evitar su degradación, aumentar la retención de humedad y fomentar un mejor uso de estos recursos. Los cambios acelerados de cobertura y uso de los suelos en el bosque tropical lluvioso afectan la productividad, la biodiversidad y las fuentes emisoras de carbono, y tienden a disminuir los compartimentos lábiles de la MOS (43). En consecuencia, estos sistemas de producción en las sabanas de la Amazonía venezolana merecen una mayor atención por su potencial para incrementar la productividad primaria y contribuir muy modestamente a contrarrestar las emisiones globales de $\mathrm{CO}_{2}$; así como en el contexto de los cambios climáticos, (18) preservar el bosque tropical lluvioso y destacar la sabana como fuente para la alimentación en esta región. Como se sabe, la pérdida de la capacidad productiva de los suelos y las elevadas concentraciones de $\mathrm{CO}_{2}$ en la atmósfera, están íntimamente relacionadas. Se ha estimado que la adopción de prácticas de manejo sustentable de los suelos, induciría un secuestro de carbono del orden de $0,9 \pm 0,3$ de C Pg año-1 ${ }^{-1}$ lo que compensaría entre un cuarto o un tercio del incremento anual de $\mathrm{CO}_{2}$ atmosférico, estimado en 3,3 C Pg año 1 (17).

La FL MOS obtenida por flotación en agua, junto a las fracciones intermedias y pasivas, representa el COS y en consecuencia las principales formas de carbono 
en los suelos ácidos tropicales carentes de carbonatos. La FL MOS sirve de indicador de los cambios en el contenido de COS inducido por distintas prácticas de manejo de los suelos $(14 ; 26)$. Por ello, los procesos de cambio de biomasa y composición florística de la vegetación, producen cambios dialécticos en el contenido y calidad de COS y la FL MOS, lo que genera a su vez, modificaciones en la biomasa y la composición florística $(38 ; 41)$.

En general estos resultados de acumulación neta de carbono atmosférico en la vegetación y el suelo, mejoran las condiciones productivas y de fertilidad. Por esta razón, la sustentabilidad de la agrícultura -animal y vegetal- en la zona intertropical esta inexorablemente ligada a la incorporación constante de insumos provenientes de la captación de la energía solar, de la lluvía, del aire y especialmente de la actividad biológica del suelo, para reducir la dependencia de los insumos agroindustriales. Los resultados aquí discutidos de acumulación de COS e incremento de la FL MOS en el suelo de la $\mathrm{SC}$, merecen atención en la perspectiva de una agricultura sustentable, con altas producciones agrícolas mediante la conversión de altas cantidades de insumos provenientes de las fuentes biológicas y atmosféricas, como es la energía solar (31).

En los últimos años el fraccionamiento físico de la MOS ha contribuido a una mejor interpretación del ciclo del carbono en los suelos. Por la resistencia o la facilidad a la descomposición de la MOS se han identificado los compartimientos activo, lento y pasivo de la MOS. Los de más facil descomposición incluyen: los residuos metabólicos y/o estructurales de plantas y animales y los primeros productos surgidos de su transformación; mientras que los com- partimientos más resistentes a la descomposición incluyen: la materia orgánica particulada y las sustancias húmicas, que han permanecido en el suelo largos periodos de transformación, entre 8-50 años y entre 400-2.200 años, respectivamente (30). Resultados recientes, utilizando espectroscopía de reflectancia difusa en el infrarrojo medio por tranformada de Fourier, han permitido identificar grupos funcionales, alifáticos y aromáticos, como indicadores de cambios asociados a las fracciones lábiles y estables de la MOS, respectivamente, en suelos tratados con abonos orgánicos e inorgánicos, de muestras provenientes de los primeros $20 \mathrm{~cm}$ del suelo (8), lo que permitió a estos autores, un enfoque sobre los mecanismos de estabilización y heterogeneidad de la MOS.

En la actualidad se requieren estudios con técnicas no destructivas, como la espectroscopía de reflectancia difusa en el infrarrojo medio por transformada de Fourier, que permitan abordar la discusión sobre qué cantidad y cualidad de COS, forma parte de las fracciones estables o lábiles de la MOS y dilucidar los factores que afectan la estabilidad de las fracciones de COS en las capas más superficiales de los epidediones. La literatura sobre secuestro de COS ha sobreenfatizado la profundidad de la muestra de suelo, por debajo de $1,5 \mathrm{~m}$, como criterio inexorable de protección física y química de las formas orgánicas en los suelos, subestimando el manejo agroecológico y los efectos de fomento y protección de la MOS en las capas más superficiales de los suelos. De acuerdo con nuestros resultados, la ganancia de COS, temporal o definitiva, estuvo más asociado al tipo de manejo y fomento agronómico, que a la profundidad de la cual provengan las muestras de suelo. 
Los cambios de fertilidad relacionados al contenido de $\mathrm{N}$ total en el suelo, mostró un bajo efecto residual de la fertilización con NPK. Los valores más altos en la SC podrían más bien asociarse a la fijación simbiótica y a su papel metabólico en el crecimiento de las especies leguminosas cultivadas in situ. El contenido de $\mathrm{P}$ total en la SC mostró diferencias significativas entre las tres profundidades y el valor más alto de $\mathrm{P}\left(147 \mathrm{mg} \mathrm{kg}^{-1}\right)$ se presentó en la profundidad intermedia. El contenido de $\mathrm{P}$ disponible resultó superior en el tratamiento SC respecto a la $\mathrm{SN}$ para todas las profundidades, lo que está en concordancia con los niveles de P total. Estos resultados reflejan el efecto residual de la fertilización fosfórica, cuatro años después de iniciado el fomento agronómico en contraste con otros autores, que no encontraron diferencias significativas entre estos tratamientos, ni una mejora de la fertilidad del suelo, por efecto residual de la roca fosfórica en muestras provenientes de un epipedón de 0-10 cm, debido al poco tiempo transcurrido después de iniciado los tratamientos (15). En este trabajo, la aplicación de roca fosfórica modificó los contenidos de $\mathrm{P}$ total y $\mathrm{P}$ disponible en la SC, pero no tuvo efecto residual sobre los valores de $\mathrm{pH}_{\text {agua }}$ y $\mathrm{pH}_{\mathrm{KCl}}$. Sin embargo, la reducción del $\mathrm{pH}_{\mathrm{KCl}}$ en la $\mathrm{SC}$, reflejó la generación de mayores lugares de superficie electronegativa y un claro desplazamiento de protones.

La literatura refiere que los restos orgánicos incorporados al suelo con altos valores $\mathrm{C} / \mathrm{N}, 50: 1$, por ejemplo, inducen una mayor inmovilización de $\mathrm{N}$, de igual forma que las relaciones $\mathrm{C} / \mathrm{P}$-orgánico, mayor o igual a 300 inducen una mayor inmovilización de $\mathrm{P}(9 ; 14)$ lo que podría interpretarse como mecanismos de compensación entre las dis- tintas fracciones del C mediante los fenómenos de mineralización e inmovilización de $\mathrm{N}$ y $\mathrm{P}$ a las distintas profundidades del suelo (35). Los menores valores para ambas relaciones $(\mathrm{C} / \mathrm{N}$ y $\mathrm{C} / \mathrm{P})$ en la $\mathrm{SC}$ muestran los cambios de fertilidad asociados a este tratamiento.

El ACP mostró una mayor explicación de la varianza asociada a un menor desplazamiento de protones $\left(\mathrm{pH}_{\mathrm{KCl}}\right)$ y a una mayor compactación del suelo (Da) en las muestras de la $\mathrm{SN}$, en contraste con el tratamiento de la $\mathrm{SC}$ a las tres profundidades de estudio, relacionada principalmente con una mayor electronegatividad coloidal (DCNC), la materia orgánica del suelo (FL MOS, COS y biomasa de raices), el $\mathrm{P}$ y el limo, cuyos efectos persistieron cuatro años después de inicado el fomento agronómico, lo que enfatizó el carácter sustentable del manejo de la SC, sin impactos adicionales de arado después de iniciado el tratamiento.

Los modelos multicausales al establecer la DCNC como variable dependiente, permitieron identificar al $\mathrm{pH}_{\text {agua }}$ y al $\mathrm{pH}_{\mathrm{KCl}}$ en el tratamiento de la SC como las variables causales con el más alto peso positivo; en contraste con la SN cuyas variables causales con el más alto peso negativo se relacionaron con el $\mathrm{pH}_{\mathrm{KCl}}$ y el Ca. Lo que podría justificar aparentemente la necesidad de la RF o de la práctica del encalado como enmiendas para elevar el $\mathrm{pH}$ en estos suelos acidos-arenosos de la $\mathrm{SN}$, que como hemos discutido tuvo un carácter efimero en el tratamiento de la $\mathrm{SC}$, y cuyos efectos sobre la DCNC, se debió a otras variables que ejercieron influencia sobre el $\mathrm{pH}_{\text {agua }} \mathrm{y}$ el $\mathrm{pH}_{\mathrm{KCl}}$, en este caso, asociadas a variables causales de naturaleza orgánica: FL MOS, COS y Da, en contraste con la SN, cuyas variables causales fueron principalmente 
de naturaleza inorgánica: limo, Da, P, N y Ca. Nosotros interpretamos que la Da tuvo un peso positivo o negativo en los modelos multicausales de la $\mathrm{SC}$ y la $\mathrm{SN}$, respectivamente, de acuerdo a los contenidos diferenciados de COS, FL MOS y arena, en ambos tratamientos.

En síntesis, el manejo con pasturas adaptadas a suelos ácidos de sabana y el fomento agronómico con roca fosfórica y NPK, indujo cambios importantes de la fertilidad del suelo, claramente asociados a los cambios de la FL MOS y el COS, que como explican los modelos teóricos-matemáticos, ejercieron una importante influencia indirecta sobre la DCNC, y ésta en la capacidad de retención de cationes y en los cambios de fertilidad, lo que es particularmente relevante en sabanas de baja fertilidad natural y drenaje interno rápido, por los efectos perdurables de estos cambios y en consecuencia por el carácter sustentable que le otorga al manejo agroecológico intensivo.

\section{CONCLUSIONES}

El fosfato de roca y las especies de pasto Urochloa dictyoneura en asociación con las leguminosas Stylosanthes capitata y Centrosema macrocarpum indujeron un incremento del COS y de la FL MOS en las capas más superficiales del suelo del orden de $743 \mathrm{~kg} \mathrm{ha}^{-1}$ año $^{-1}$ de COS. Los resultados muestran que el tratamiento SC indujo cambios químicos y fisicoquímicos perdurables cuatro años después de establecidas las pasturas. Los primeros $10 \mathrm{~cm}$ del suelo de la SC fueron significativamente modificados por el manejo agronómico, que a su vez, indujo cambios en el tiempo, de los atributos de fertilidad como: la minerali- zación de la materia orgánica depositada en el suelo y los fenómenos de naturaleza electroquímica que generan nuevos sitios de intercambio catiónico.

Se demuestra el incremento en la fijación de COS en el suelo y la potencialidad de aprovechamiento agrícola sustentable que tienen estos agroecosistemas, mediante el manejo agroecológico intensivo de los suelos ácidos-arenosos, de cargas variables dependientes del $\mathrm{pH}$, lo cuales tienen una amplia distribución en las sabanas venezolanas de buen drenaje. Estos resultados enfatizan la intensificación sustentable del uso de estas sabanas en la Amazonía venezolana, para contribuir a reducir la presión sobre el bosque tropical lluvioso, a través del fomento agronómico de pasturas adaptadas y el pastoreo con ovejos en estos ecosistemas.

\section{AGRADECIMIENTOS}

Los autores agradecen el financiamiento otorgado por el Conicit, a través del Proyecto RP VII 290089 y al Consejo de Desarrollo Científico y Humanístico de la Universidad Central de Venezuela a través del proyecto $\mathrm{N}^{\circ}$ 03.31.4642.2000. De igual modo, al Ing. Agro. Frans Torres, al Dr. Antonio Sánchez y a la comunidad Guahiba de Sabaneta de Guayabal, por su valiosa contribución en la génesis e implementación de esta experiencia. Los autores agradecen al Dr. Roschman González su trabajo en el tratamiento estadístico de los resultados. 


\section{REFERENCIAS}

1.- ALLEN, S.E.; GRIMSHAW, H.M.; PARKINSON, J.A. Y QUARMBY, C. 1974. Chemical analysis of ecological materials. John Wiley and Sons, New York. p. 565. ISBN 100632003219 .

2.- ANDERSON, J. Y INGRAM, J. S. 1993. Tropical soil biology and fertility: A handbook of methods. C. A. B. International. p. 221. https://doi.org/10.1017/ S0014479700024832

3.- ARAUJO, Y. Y LÓPEZ-HERNÁNDEZ, D. 1999. Earthworm Populations in a Savanna Agroforestry System of Venezuelan Amazonia. Biol. Fert. Soils 29:413-418

4.- BLANCANEAUX, PH.; HERNÁNDEZ, S. Y ARAUJO, J. 1977. Estudio edafológico preliminar. Sector Puerto Ayacucho, Territorio Federal Amazonas. Serie Informes Científicos. DGIIA10/01MARNR, Caracas. p. 120. https:/www.researchgate.net/profile/Philippe_Blancaneaux/publication/281416145

5.- CASANOVA, E. 1996. Introducción a la ciencia del suelo. Universidad Central de Venezuela. Consejo de Desarrollo Científico y Humanístico. Caracas. p. 379.

6.- CIAT (Centro Internacional de Agricultura Tropical). 2012. Annual report 1999. Project IP-5. Tropical grasses and legumes: Optimizing genetic diversity for multipurpose use. p. 175. http://ciat-library.ciat.cgiar.org/Articulos_Ciat/2015/Project_IP-5_Annual_Report_1999.pdf

7.- DECAËNS, T., J.H. GALVIS Y E. AMÉZQUITA. 2001. Properties of the Structures Created by Ecosystem Engineers on the Soil Surface of a Colombian Savanna. Chapter 11. 151-175. En: J.J Jiménez y R. Thomas (Eds.) Nature's Plow: Soil Macroinvertebrate Communities in the Neotropical Savannas of
Colombia. Centro Internacional de Agricultura Tropical (CIAT). Cali, Colombia. https:// books.google.co.ve/books?id=-uLuarMugHIC\&pg=PA209\&lpg=PA209\&dq=Decaëns, +T.,+J.H.+Galvis+y+E.+Amézquita. +2001 .

8.- DEMYAN, M.S.; RASCHEA, F.; SCHULZB, E.; BREULMANNB, M.; MÜLLERC, T. Y CADISCHA, G. 2012. Use of specific peaks obtained by diffuse reflectance Fourier transform mid-infrared spectroscopy to study the composition of organic matter in a Haplic Chernozem. European Journal of Soil Science. 63(2):189-199. https://doi.org/10.1111/j.1365-2389.2011.01420.x

9.- ENWEZOR, W.O. 1968. Significance of the C:organic-P ratio in the mineralization of soil organic phosphorus. Soil Sci. 100 (1):61-65.

10.- FAO. 2017. Carbono Orgánico del Suelo: el potencial oculto. Organización de las $\mathrm{Na}-$ ciones Unidas para la Alimentación y Agricultura. Roma, Italia p. 90 http://www.fao. org/3/b-i6937s.pdf

11.- FILSER, J.; FABER J.; TIUNOV A.; BRUSSAARD, L.; FROUZ, J.; DEDEYN, G; UVAROV, A.; BERG M.; LAVELLE, P.; LOREAU, M.; WALL, D.; QUERNER, P.; EIJSACKERS, H.; JIMÉNEZ, J.J. 2016. Soil fauna: key to new carbon models. Soil, 2, 565-582, 2016. www.soil-journal. net/2/565/2016/ doi:10.5194/soil-2-565-2016.

12.- GARCÍA, P. 1994. Los suelos del estado Amazonas: sus potencialidades agrícolas. Venesuelos. 2(2): 59-66.

13.- GRACE, J.; SAN JOSÉ, J.J.; MEIR, P.; MIRANDA, H. Y MONTES, R. 2006. Productivity and carbon fluxes of tropical savannas. J. Biogeogr.33: 387-400. http:// citeseerx.ist.psu.edu/viewdoc/download?doi $=10.1 \cdot 1.475 .1824 \&$ rep $=$ rep $1 \&$ type $=$ pdf 
14.- GALANTINI, J.; IGLESIAS, J.; LANDRISCINI, M.; SUÑER, L. Y MINOLDO, G. 2008. Calidad y dinámica de las fracciones orgánicas en sistemas naturales y cultivados. pp. 71-95. En: Estudio de las fracciones orgánicas en suelos de la Argentina. (Galantini, J., Editor; Suñer, L.; Landriscini, M. e Iglesia, J. Compiladores) Editorial de la Universidad Nacional del Sur. Bahía Blanca, Argentina. pp. 308.

15.- HERNÁNDEZ-VALENCIA, I.; LÓPEZ, A. Y LÓPEZ-HERNÁNDEZ, D. 1999. Cambios en los contenidos nutricionales en suelos arenosos de sabanas del Amazonas bajo fertilización orgánica prolongada. Ecotropicos. 12(1): 9-14. https://www.researchgate.net/profile/Ismael_Hernandez-Valencia/ publication/233898460.

16.- KANE, D. 2015. Carbon Sequestration Potential on Agricultural Lands: A Review of Current Science and Available Practices, s.1.: s.n. https://sustainableagriculture.net/ wp-content/uploads/2015/12/Soil_C_review_Kane_Dec_4-final-v4.pdf

17.- LAL, R. 2004. Soil carbon sequestration to mitigate climate change. Geoderma. 123:1-22. https://doi.org/10.1016/j.geoderma.2004.01.032

18.- LAL, R. 2004. Soil carbon sequestration impacts on global climate change and food security. Science.304: 1623-1627. https://www.cagg.org/wp-content/uploads/1623.pdf

19.- LEÓN, S.T. 2005. La agricultura ecológica como posición política frente al actual modelo de desarrollo agrario colombiano. Acta Biológica Colombiana. 10:7:67-73. https://www. redalyc.org/pdf/3190/319028576005.pdf

20.- LÓPEZ-HERNÁNDEZ, D. 2010. Agricultural systems in the savanna-forest ecotone of Venezuelan Amazonian. Evaluation of soil quality indicators. Chapter 1. En: Amazon Basin: Plant Life, Wildlife and Environment. Environmental Research Advances Series.
Nicolas Rojas y Rafael Prieto (eds). Nova Science Publishers Inc. p. 1-45. https://www. researchgate.net/publication/276276388

21.- MAHANEY, W.M.; SMEMO, K.A. Y GROSS, K. L. 2008. Impacts of C4 grass introductions on soil carbon and nitrogen cycling in C3 dominated successional systems. Oecologia. 157: 295. https://doi.org/10.1007/ s00442-008-1063-5

22.- MEDINA, E. 1996. Biodiversity and Nutrient Relations in Savanna Ecosystems: Interactions Between Primary Producers, Soil Microorganisms, and Soils. p. 45-57. En: Solbrig O.T., Medina E., Silva J.F. (eds) Biodiversity and Savanna Ecosystem Processes. Ecological Studies (Analysis and Synthesis), vol 121. Springer, Berlin, Heidelberg. p. 224. https://link.springer.com/chapter/10.1007/978-3-642-78969-4_3

23.- MOHR, E.C.J.; VAN BAREN, F.A. Y VAN SCHUYLENBERGH, J. 1972. Tropical soils: A comprehensive study of their genesis. Third edition, Mouton-Ichtiar Baruvan Hoeve. The Hague-Paris-Djakarta. p. 481.

24.- MURPHY, J. Y RILEY, J. 1962. A modified single solution method for the determination of phosphate in natural waters. Anal. Chim. Acta. 27:31-36. https://www.academia.edu/3720851

25.- OJEDA-FALCÓN, A.D. 2015. La intensificación de la agricultura y el modelo eco-productivo socialista en Venezuela. Tiempos para pensar Investigación social y humanística hoy en Venezuela, Tomo I (Compiladora Alba Carosio). Consejo Latinoamericano de Ciencias Sociales (CLACSO). Fundación Centro de Estudios Latinoamericanos Rómulo Gallegos, Caracas, Venezuela. pág 385-394. ISBN 978-980-399-069-5. https:// www.academia.edu/35012022/ALBA_CAROSIO_compiladora 
26.- OJEDA-FALCÓN, A.D. Y LÓPEZ-HERNÁNDEZ, D. 1992. Changes of phosphorus contents in light and heavy soil organic matter induced by different tillage systems. 1994. p. 673-678. En: Senesi N y Miano TM (eds.) Humic substances in the global environment and implications in human health, 6th international meeting, international humic substances society, september 20-25. Istituto di chimica agraria, Università di Bari, Monopoli, Italy. ISBN: ISBN 0444895930.

27.- OJEDA-FALCÓN, A.D.; STEIN, M. Y LÓPEZ-HERNÁNDEZ, D. 2009. Secuestro de carbono orgánico y cambios de fertilidad en un ultisol de sabanas en la Amazonía venezolana. Bioagro. 21(3): 195-202. http://www. ucla.edu.ve/bioagro/Rev21(3)/7.\%20Secuestro\%20de\%20carbono\%20orgánico.pdf

28.- OLSEN, S.R.; COLE, C.V.; WATANABE, F.S. Y DEAN, L.A. 1954. Estimation of available phosphorus in soils by extractions with sodium bicarbonate. USDA. Circ. $p$. 939. https://archive.org/details/estimationofavai939olse/page/2

29.- ORTIZ-PULIDO, R. 2000. Análisis de ruta en biología: Estadística para sistemas multicausales. Interciencia. 25(7): 329-336. https://es.scribd.com/document/318523492

30.- PARTON, W.J.; SCHIMEL, D.C.; COLE, C.V. Y OJIMA, D.S. 1987. Analysis of factors controlling soil organic matter levels in the Great Plains grasslands. Soil Sci.Soc. Am. J. 51:1173-1179. http://dx.doi.org/10.2136/ sssaj1987.03615995005100050015x

31.- PINHEIRO, L.C. 2004. Pastoreo racional Voisin. Tecnología agroecológica para el tercer milenio. Editorial Hemisferio Sur. p. 253.

32.- RAMIA, M. 1967. Tipos de sabana en Los Llanos de Venezuela. Bol. Soc. Ven. Cien. Nat. 27:264-288.
33.- RAO, I.M.; AYARZA, A.; THOMAS, R.J.; FISHER, M.J.; SANZ, J.I.; SPAIN, J.M. Y LASCANO, C.E. 1992. Soil-Plant Factors and Processes Affecting Productivity in Ley Farming. Chapter 9. p. 145-175. Pastures for the Tropical Lowlands. Centro Internacional de Agricultura Tropical (CIAT), Colombia. p. 238. http://ciat-library.ciat. cgiar.org/articulos_ciat/2013/42445_Soil plant_factors_and_processes_affecting_productivit.pdf

34.- RONDÓN, M.A.; ACEVEDO, D.; HERNÁNDEZ, R.M.; RUBIANO, Y.; RIVERA, M.; AMEZQUITA, E.; ROMERO, M.; SARMIENTO, L.; AYARZA, M.; BARRIOS, E. Y RAO, I. 2006. Carbon sequestration potential of the neotropical savannas of Colombia and Venezuela. En: Carbon sequestration in soils of Latin America. Lal, R.; Cerri, C.; Bernoux, M.; Etchevers, J. y Pellegrino, C. (eds.) Haworth Press Inc. New York. p. 213-243. ISBN 9781560221371.

35.- SUÑER, L.; GALANTINI, J. Y ROSELL, R. 2008. Transformaciones del fósforo en las fracciones orgánicas en suelos naturales y cultivados. pp. 181-193. En: Estudio de las fracciones orgánicas en suelos de la Argentina. (Galantini, J., Editor; Suñer, L.; Landriscini, M. e Iglesia, J. Compiladores) Editorial de la Universidad Nacional del Sur. Bahía Blanca, Argentina. pp. 308.

36.- SZCZERBAN, E. 1974. Geología y petrología del área de Puerto Ayacucho, Territorio Federal Amazonas, Venezuela. Unidad de Geología. MOP. CODESUR Caracas. p. 243

37.- THENG, B.K.G. 1980. Soils with variablecharge. Soils Bureau, Department of Scientific and Industrial Research. New Zealand Society of Soil Science. Private Bag. Lower Hutt. p. 17-34. p.448. ISBN 10: 0477066682 
38.- TILMAN, D. 1988. Plant strategies and dynamics and structure of plant communities. Monographs in Population Biology-26. Princeton University Press. p. 376. ISBN9780691084893

39.- UEHARA, G. Y GILLMAN, G. 1981. The mineralogy chemistry and physics of tropical soils with variable charge clays. Westview Tropical Agricultural Series, No 4, Westview Press, Boulder, Colorado, USA. p.170. ISBN 0891584846

40.- UNEP. 2010. United Nations environment programme (UNEP). Global environment outlook (GEO): Latin American and the Caribbean (LAC). Global Environment Outlook GEO LAC 3. p. 380. ISBN: 978-92-8072955-9.

41.- VACCARO, S.; ARTURI, M.F.; GOYA, J.F.; FRANGI, J.L. Y PICCOLO, G. 2003. Almacenaje de carbono en estadios de la sucesión secundaria en la provincia de misiones Argentina. Interciencia. 28(9): 521527. https://www.researchgate.net/publication/237033032
42.- VOISIN, A. 1960. Dynamique des herbages. La Maison Rustique, Paris: XVI. p. 319. https://lib.ugent.be/catalog/rug01:000929089

43.- WOOMER, P.L.; MARTIN, A.; ALBRECHT, A.; RESCK, D.V.S. Y SCHARPENSEEL, H.W. 1994. The importance and management of soil organic matter in the tropics. p. 47-80. En: Woomer, P. L. y Swift, M. J. (eds.). The Biological Management of Tropical Soil Fertility. A Wiley-Sayce Publication. p. 243. http://horizon.documentation.ird.fr/exl-doc/pleins_textes/pleins_textes_7/b_fdi_55-56/010021562.pdf

44.- ZAPATA, R. 2004. Química de la acidez del suelo. Medellin, Colombia pp 208. http://bdigital.unal.edu.co/1735/1/9583367125.1.pdf 